\title{
Recommendations for the measurement of the QT interval during the use of drugs for COVID-19 infection treatment. Updatable in accordance with the availability of new evidence
}

\author{
Enrique Asensio ${ }^{1} \cdot$ Rafael Acunzo ${ }^{2} \cdot$ William Uribe $^{3} \cdot$ Eduardo B. Saad $^{4} \cdot$ Luis C. Sáenz $^{5}$
}

Received: 3 April 2020 / Accepted: 23 April 2020 / Published online: 16 May 2020

(C) Springer Science+Business Media, LLC, part of Springer Nature 2020

\begin{abstract}
COVID-19 infection has shown rapid growth worldwide, and different therapies have been proposed for treatment, in particular, the combination of immune response modulating drugs such as chloroquine and hydroxychloroquine (antimalarials) alone or in combination with azithromycin. Although the clinical evidence supporting their use is scarce, the off label use of these drugs has spread very quickly in face of the progression of the epidemic and the high mortality rate in susceptible populations. However, these medications can pathologically prolong the QT interval and lead to malignant ventricular arrhythmias such that organized guidance on QT evaluation and management strategies are important to reduce morbidity associated with the potential large-scale use.
\end{abstract}

Keywords Hydroxychloroquine · Chloroquine · Azithromycin · QT interval · Torsade de pointes · COVID 19

COVID-19 infection has shown rapid growth worldwide, and different therapies have been proposed for treatment, especially in critical patients with acute respiratory failure [1]. One of these therapeutic strategies suggests the use of immune response modulating drugs such as chloroquine and hydroxychloroquine (antimalarials) alone or in combination with azithromycin (macrolide antibiotic); the use of antivirals such as ritonavir and lopinavir have also been suggested. Although the clinical evidence supporting their use is scarce and mostly limited to in vitro studies, the off label use of these drugs as first-line therapy and even as prophylaxis has spread very quickly in face of the progression of the epidemic and the high mortality rate in susceptible populations. However, it is very important to note that all of these medicines can pathologically prolong the QT interval in patients at high risk by genetic predisposition (with

Luis C. Sáenz

lcsaenz@cardioinfantil.org

Division of Internal Medicine, Hospital H, Querétaro, Mexico

2 Division of Cardiology, Hospital Ramos Mejía, Buenos Aires, Argentina

3 CES Cardiología, Medellín, Colombia

4 Arrhythmias Service, Hospital Pró-Cardíaco, Río de Janeiro, Brazil

5 International Center of Arrhythmias, Fundación CardioInfantill-Instituto de Cardiología, Bogotá, Colombia congenital long QT syndrome or in patients with polymorphisms but without phenotypic expression) or those with acquired predisposition. This risk may be further increased by the wide interaction of these drugs with countless medications such as antibiotics, antiarrhythmics, anesthetics, and muscle relaxants, among others (http://www.covid19-druginteractions. org/). For these reasons, an increase in the occurrence of malignant arrhythmias such as "torsade de pointes" or ventricular fibrillation with indiscriminate use of these drugs is expected if preventive measures are not established (Fig. 1).

LAHRS in collaboration with the Colombian College of Electrophysiology and the Argentine, Brazilian, and Mexican Societies of Cardiac Electrophysiology (SADEC, SOBRAC, SOMEEC) wish to contribute to the mitigation of the risk of occurrence of this type of arrhythmias by the elaboration of this practical document on how to measure the QT interval, their normal values and ranges in which there is true risk associated with QT prolongation [2-4] in order to help identify not only patients with long QT at baseline but also those at risk of further abnormal prolongation in response to the initiation of therapy for COVID-19 [5-7] (Table 1). In addition, the algorithm recently proposed by the Mayo Clinic [8] for decision-making during the use of hydroxychloroquine and azithromycin has been adapted in patients with COVID-19, with respect to QTc (Table 2). Links of interest have been added where you will find information about the interactions of these medicines, as well as automated formulas for QT correction. We hope that this additional practical material will contribute to the minimization of face-to-face 
"Torsade de pointes" (tip-twisted, helical tachycardia) in the Holter of a patient receiving quinidine who had high-grade $A V$ block and syncope.

The amplitude, polarity and configuration of QRS complexes vary continuously and generate a sinusoidal image around the isoelectric line. Although "torsades de pointes" usually presents as self-limited paroxysms, degeneration to ventricular fibrillation can also occur.
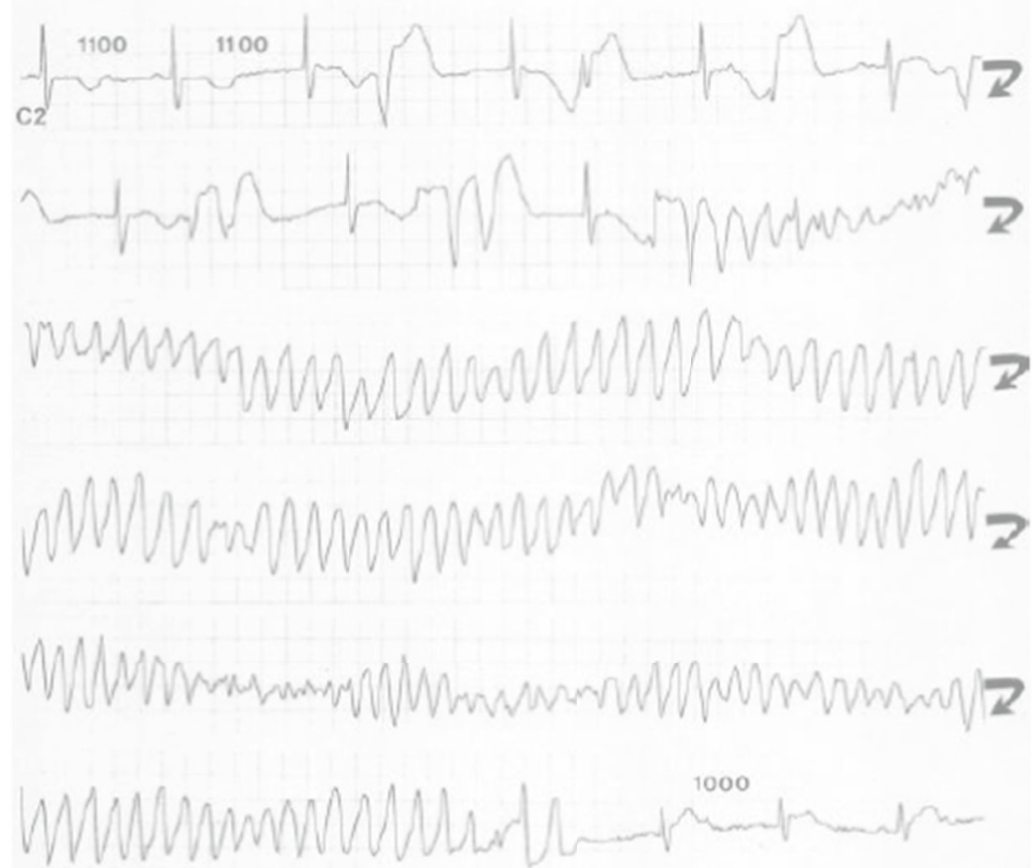

Fig. 1 Torsade de pointes

Table 1 Modifiable and non-modifiable risk factors for inducible QT prolongation and risk of "torsade de pointes"/ventricular fibrillation

Modifiable risk factors

- Electrolyte abnormalities:

Hypocalcemia $(<4.65 \mathrm{mg} / \mathrm{dl})$

Hypokalemia $(<3.4 \mathrm{mmol} / \mathrm{L})$

Hypomagnesemia $(<1.7 \mathrm{mg} / \mathrm{dl})$

- Drugs that prolong the QT

Simultaneous use of $\geq 1$ drug

- Starvation/anorexia nervosa

Non-modifiable risk factors

- Common comorbidities:

Acute coronary syndrome

Bradyarrhythmia with $\mathrm{HR}<45 \mathrm{bpm}$

Decompensated heart failure $(\mathrm{EF}<40 \%)$

Congenital long QT syndrome

Renal failure on dialysis

Diabetes mellitus

Hyperthrofic cardiomyopathy

Hypoglycemia

Pheochromocytoma

Post cardiac arrest (1st $24 \mathrm{~h}$ )

Status post syncope or convulsion (1st $24 \mathrm{~h}$ )

Cerebrovascular event, subarachnoid hemorrhage, or cranial trauma (1st 7 days)

- Personal or familial previous history:

Previous QT prolongation or unexplained sudden death

- Demographic factors:

Age $>65$ years

Female sex

Modified from: Giudicess et al in Mayo Clinic Proceedings 2020 (published online $03 / 25 / 2020$ ) consultation and help to reduce the risk of contagion for patients, hospital staff, and their families, as well as to prioritize the use of personal protection equipment for the first-line care of infected patients.

\section{QT interval measurement, correction, and normal values}

The measurement of QT (QTm) and corrected QT (QTc) intervals should be routine in the electrocardiographic evaluation.

Table 2 Calculation of risk score for QTc interval prolongation

\begin{tabular}{lc} 
Risk factors points & Points \\
\hline Age $\geq 68$ years & 1 \\
Female sex & 1 \\
Loop diuretic & 1 \\
Serum $\mathrm{K}^{+} \leq 3.5 \mathrm{mEq} / \mathrm{L}$ & 2 \\
Admission QTc $\geq 450 \mathrm{~ms}$ & 2 \\
Acute myocardial infarction & 2 \\
$\geq 2$ QTc-prolonging drugs & 3 \\
Sepsis & 3 \\
Heart failure & 3 \\
One QTc-prolonging drug & 3 \\
Maximum risk score & 21 \\
\hline
\end{tabular}

A score of $\leq 6$ predicts low risk, 7-10 medium risk, and $\geq 11$ high risk of drug-associated QT prolongation. Modified from Tisdale et al [9]. in Circ Cardiovasc Qual Outcomes. 2013;6(4):479-487 
Fig. 2 QT measurement and formula for correction

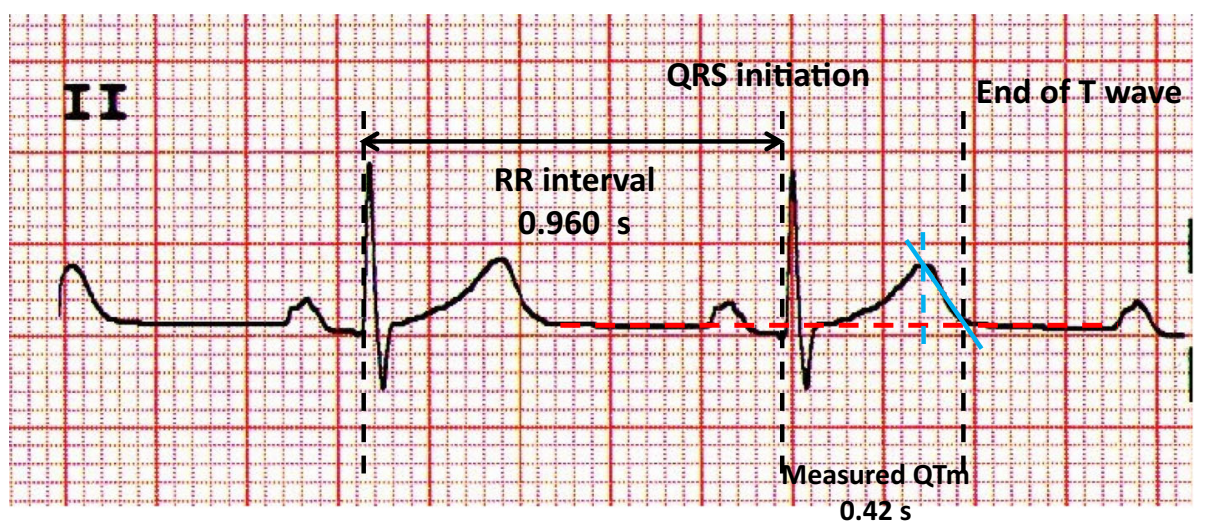

The QT interval is measured from the start ofthe QRS to the end ofthe T wave (QTm).

- To determine the end ofthe T wave, a line is drawn from its vertex (blue dotted line) following the slope ofits descending inscription (full blue line) to where itintersects the baseline (dotted line in red).

- Ideally, the QT should be measured in leads with Q waves DII and V5.

\section{QT Correction with Bazett's Formula:}

$$
\frac{\mathrm{QTm}}{\sqrt{\mathrm{RR}}} \frac{0.42}{\sqrt{0.96}} \Rightarrow \frac{0.42}{0.9797}=0.428
$$

The QT interval is the time period between the beginning of the activation of the ventricular myocardium and the end of its repolarization that are represented, respectively, by the beginning of the QRS and the end of the T wave (Fig. 2). The end of the $\mathrm{T}$ wave in turn is determined as the point of return of the wave to the isoelectric line drawn between the $\mathrm{T}$ and $\mathrm{P}$ waves (Fig. 2).

As a standard recommendation, evaluating the QT in a 12lead ECG is taken with paper speed at $25 \mathrm{~mm} / \mathrm{s}$ and $10 \mathrm{~mm} /$
$\mathrm{mV}$ amplitude, and measuring is done in leads II and V5 or V6, from which the longest value measured is taken.

Sometimes the $U$ wave may be observed after the $T$ wave (Fig. 3). If the U wave is sufficiently separated from the end of the T wave, the QT interval can be measured without difficulty as described (excluding the U wave). Conversely, when the U wave is inscribed at the end of the $\mathrm{T}$ wave, it is difficult to differentiate it from notched $\mathrm{T}$ waves, affecting the accuracy of the QT measurement. The notched T wave is recognized by
Fig. 3 Measuring QT in presence of $U$ wave
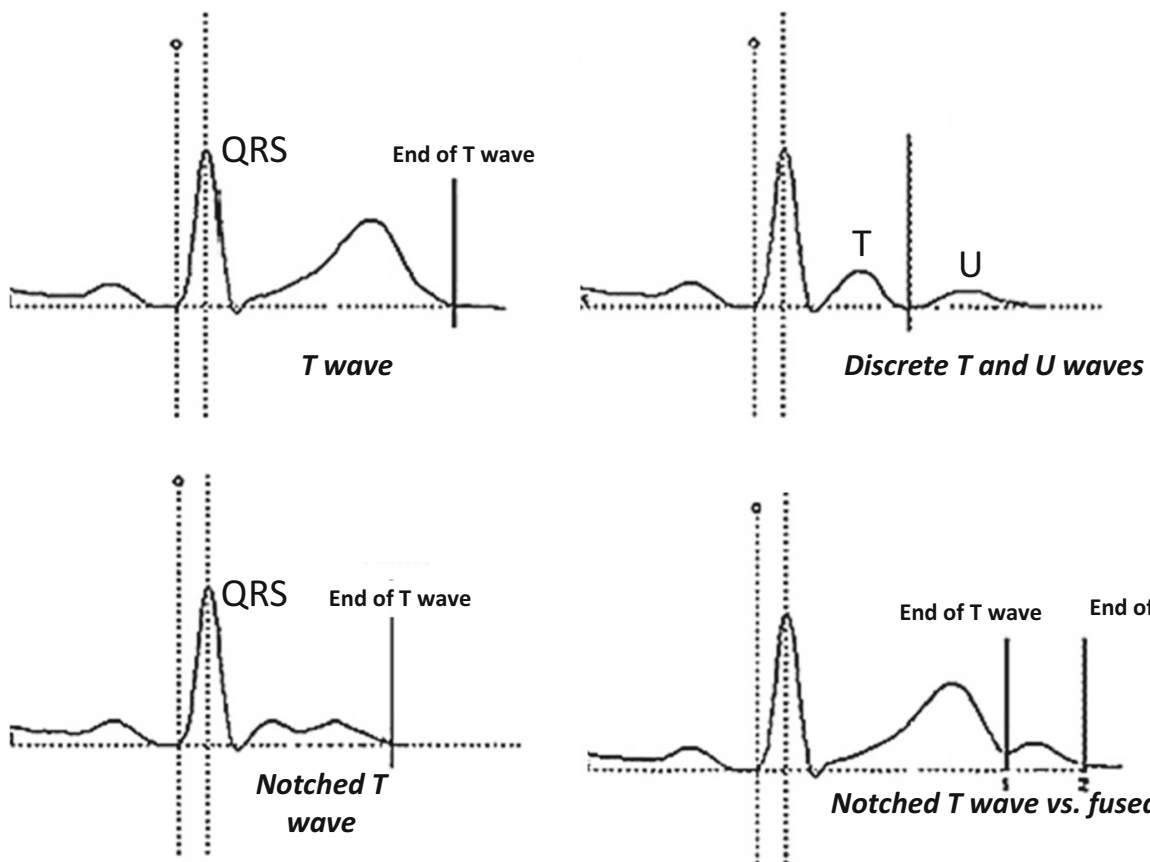
the presence of two waves of the same amplitude that are fused without return to the baseline (Fig. 3). In these cases, the QT measurement should be done to the point where the downward inscription of the second wave crosses the baseline (Fig. 3). In cases where the $\mathrm{T}$ wave has two components but the second one is much smaller in amplitude than the first, it is difficult to differentiate whether that is a notched $\mathrm{T}$ wave or $\mathrm{U}$ wave inscribed at the end of the T wave. In the latter case, it is suggested to measure the QT interval from the start of the QRS to the nadir between the two components (where they merge) and also report the QTU interval measured to the point at which the second component crosses the baseline (Fig. 3).

QT must also be corrected to the heart rate, and there are several formulas to do so. However, the most commonly used is the Bazett's. This formula includes the measured QT value (QTm in seconds) and the interval between the previous QRS and the QRS in which the QT is measured (RR interval in seconds) (Fig. 2). Some calculators allow automated correction of QT if the average heart rate is included (https:// reference.medscape.com/calculator/qt-interval-correctionekg).

QTc values considered to be normal (which do not exceed the 99th percentile value for healthy individuals) vary by gender and age (Table 3). As a general reference, patients with QTc at rest with prolongation $\geq 500 \mathrm{~ms}$, regardless of cause (congenital vs. acquired), is considered a risk marker for "torsade de pointes" or ventricular fibrillation.

Other formulae to correct the QT interval for heart rate have been proposed (Table 4). A single-center retrospective study compared the available formulae in a hospital population of patients older than 19 years that were in sinus rhythm, which showed narrow QRS and a heart rate $<90 \mathrm{bpm}$. The Fridericia and Framingham formulae showed better rate correction and improved prediction of 30-day and 1-year mortality when compared with the Bazett formula that seemed to overestimate the QTc. However, those formulae have not been prospectively compared in a wider population with abnormal heart rhythms or ventricular conduction defects. In any case, the intra-individual changes comparing control with basal QTc after the first doses of a QT prolonging medication are of key importance to identify patients in risk for "torsade de

Table 3 Normal values for corrected QT by age group and gender

QTc interval values by age and gender (seconds)

\begin{tabular}{lccc}
\hline & Under 15 years & Adults & \\
\hline & Both genders & Women & Men \\
Normal & $0.35-0.44$ & $0.35-0.45$ & $0.35-0.43$ \\
"Borderline" & $0.44-0.46$ & $0.45-0.47$ & $0.43-0.45$ \\
Prolonged & $>/=0.47$ & $>/=0.48$ & $>/=0.46$ \\
\hline
\end{tabular}

Table 4 QT Correction for heart rate (Published formulae)

\begin{tabular}{ll}
\hline Bazett & $\mathrm{QTcB}=\mathrm{QT} / \mathrm{RR}^{1 / 2}$ \\
Fridericia & $\mathrm{QTcFr}=\mathrm{QT} / \mathrm{RR}^{1 / 3}$ \\
Framingham & $\mathrm{QTcFra}=\mathrm{QT}+0.154(1-\mathrm{RR})$ \\
Hodges & $\mathrm{QTcH}=\mathrm{QT}+0.00175([60 / \mathrm{RR}]-60)$ \\
Rautaharju & $\mathrm{QTcR}=\mathrm{QT}-0.185(\mathrm{RR} 1)+\mathrm{k}(\mathrm{k}=+0.006 \mathrm{~s}$ \\
& for men and $+0 \mathrm{~s}$ for women $)$ \\
\hline
\end{tabular}

pointes"/ventricular fibrillation associated with pathologic prolongation of the QT interval (called "QTc reactors" by Giudicessi et al.).

In patients with $\mathrm{AF}$ and variable rate response, the correction of the QT for heart frequency could be problematic [10]. In those cases, the "RR" in existing QT correction formulas (measured in seconds as the interval between the previous QRS and the QRS in which the QT is measured) is replaced with a weighted average of the previous $5 \mathrm{RR}$ intervals. This method to correct the QT has shown to improve the ability of the formulae to normalize QT intervals in AF patients.

In patients with wide QRS secondary to branch blocks, paced rhythm, pre-excitation by an accessory pathway, use of class $1 \mathrm{C}$ antiarrhythmic drugs, or advanced heart disease, the duration of QT can be prolonged by the increase in myocardial activation time rather than by prolonging repolarization. In these cases, the QTc must be adjusted to the QRS duration using the following formula: [QTc adjusted to QRS width $=\mathrm{QTc}-(\mathrm{QRS}-100 \mathrm{~ms})]$. For example, if the QRS is $180 \mathrm{~ms}, 180-100=80 \mathrm{~ms}$ and these $80 \mathrm{~ms}$ are subtracted from the conventionally measured QTc value which for the example would be $520 \mathrm{~ms}$. In this way, the final value of QTc adjusted to the duration of the QRS would be $520-80 \mathrm{~ms}=$ $440 \mathrm{~ms}$, in reference to which the therapeutic decision should be made.

\section{Decision algorithm for the use of hydroxychloroquine and azithromycin for the treatment of patients with COVID-19 infection in relation to QTc (adapted from the proposed by the Mayo Clinic) (Fig. 4)}

We have adapted for this document the algorithm proposed by the Mayo Clinic with the aim of serving as a practical guide for the use of hydroxychloroquine and azithromycin in COVID-19 infection with respect to the behavior of QT and the presence of risk factors that prolong it. It should be noted that the indication for the use of these medicines should be defined depending on the protocols formulated by international and local regulatory entities [11], as well as by each institution. Depending on future changes in the indications for their use, as well as the experience gained from their safety 


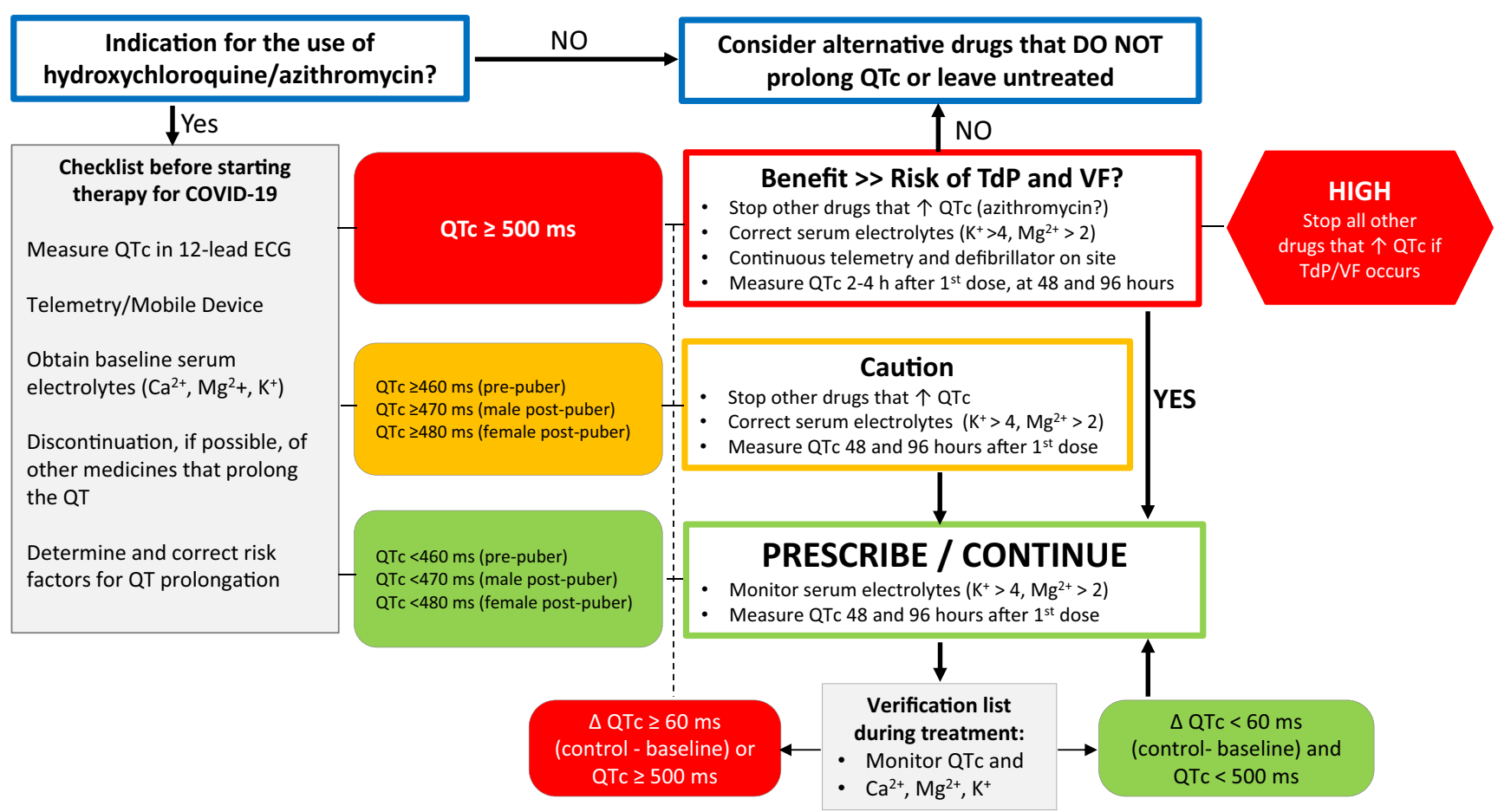

Fig. 4 Decision algorithm for the use of hydroxychloroquine with/without concomitant use of azithromycin in COVID-19 infection

profile during the pandemic, the proposed algorithms may be modified. Special updates should be expected regarding indications for outpatient initiation vs. intrahospital administration of these drugs and the type of electrocardiographic monitoring with which the QT should be measured before and during administration (12 lead ECG, continuous telemetry, devices for remote monitoring [12] or without indication for follow-up measurement).

1. Once it is decided that the patient has an indication for the use of these drugs, it is suggested that a baseline checklist is followed that includes:

a. QTc measurement

a. Baseline evaluation of serum electrolytes

b. Interruption of other nonessential drugs that can also prolong the QT (https://www.crediblemeds.org/)

c. Identification of risk factors associated with QT prolongation (Table 1).

The identification and correction, if possible, of risk factors for inducible QT prolongation are essential to prevent "torsade de pointes"/ventricular fibrillation appearance. Those risk factors have been included by Tislade et al. in a risk score to predict QT interval prolongation in hospitalized patients that also could be used to guide the administration of COVID medications (Table 2).

2. The QTc interval measurement can be done on the 12-lead surface ECG as described in the previous paragraphs.
However, taking ECG as standard can increase the risk of contact contagion for patients, health workers, and their families. For this reason and in accordance with local regulations, QTc measurement can be done in telemetry ECG leads (one or more) during continuous monitoring in hospitalized patients or by mobile systems according to their local availability and regulation.

3. If the baseline QTc is below the 99th percentile for gender and age (highlighted in green in the algorithm), the COVID-19 medicines may be started.

a. Quickly, if the QT interval measured in 12-lead ECG or a telemetry or mobile device is less than half of the preceding RR interval, the calculated QTc will always be $<460 \mathrm{~ms}$, and therefore, it is safe to start the drug for COVID-19.

4. In patients with $\mathrm{QTc} \geq 500 \mathrm{~ms}$ (highlighted in red in the algorithm), initiation of these medications should be decided according to a balance between the expected benefit (risk of onset/progression of acute respiratory failure) and the risk of malignant ventricular arrhythmias associated with QT prolongation (depends on the QTc value in addition to the presence of risk factors).

a. In addition to the points suggested on the initial checklist, continuous monitoring of the patient's rhythm in units with immediate defibrillation capacity is suggested, due to increased risk of malignant arrhythmias. 
b. In these patients with baseline QTc $\geq 500 \mathrm{~ms}$, hydroxychloroquine use alone is suggested thus avoiding concomitant use of azithromycin.

c. In the presence of $\mathrm{TdP} / \mathrm{VF}$, all medicines associated with QT prolongation should be discontinued.

5. In patients with baseline QT $<500 \mathrm{~ms}$ but increased to $\geq$ $500 \mathrm{~ms}$ or in whom the QTc delta (QTc control minus baseline QTc) is $\geq 60 \mathrm{~ms}$ after combined use of hydroxychloroquine and azithromycin, suspension of the latter drug is suggested.

6. The frequency with which QTc should be measured will depend on the pharmacokinetics of these drugs, baseline QTc, and associated risk of TdP/VF and the method used for ECG monitoring and the consequent risk of contagion during evaluation (Fig. 4). Of note, the US Food and Drugs Administration (FDA) has approved the use of KardiaMobile 6 L (AliveCore) to monitor the QTc duration in COVID-19 patients receiving QT-prolonging drugs. This avoids exposure of ECG technicians to affected patients and unnecessary use of PPE.

\section{References}

1. Driggin E, Madhavan M, Bikdeli B, Chuich T, Laracy J, Bondi G, et al. Cardiovascular considerations for patients, health care workers and health system during the coronavirus disease 2019 (COVID-19) pandemic. J Am Coll Cardiol March. 2020. https:// doi.org/10.1016/j.jacc.2020.03.031.

2. Golderberg I, Moss A, Zareba W. QT interval: how to measure it and what is "normal". J Cardiovasc Electrophysiol. 2006; 17:333-6.

3. Johnson J, Ackerman M. QTc: how long is too long? Br J Sports Med. 2009;43(9):657-62.
4. Hocht C, Opezzo J, Taira C. Intervalo QT prolongado inducido por fármacos desde el punto de vista de un farmacólogo. Rev Arg Cardiol. 2004;72(6):474-80.

5. Woosley R. Cardiac complications of chloroquine based therapy. Cardiology. 2020. https://www.practiceupdate.com/content/ cardiac-complications-of-chloroquine-based-therapy/98054.

6. CDC Therapeutic options for patients with COVID 19. https:// www.cdc.gov/coronavirus/2019-ncov/hcp/therapeutic-options. $\mathrm{html}$.

7. Choi Y, Hong L, Chung D, Choi J, Yoon D. Risk evaluation of azithromycin- induced QT prolongation in real-world practice. Biomed Res Int. 2018;2018:1-8. https://doi.org/10.1155/2018/ 1574806 .

8. Giudicessi J, Noseworhty P, Friedman P, Ackerman M. Urgent guidance for navigating and circumventing the QTc prolonging and torsadogenic potential of possible pharmacotherapies for COVID-19. Mayo Clin Proc. 2020;95.

9. Tisdale JE, Jaynes HA, Kingery JR, Mourad NA, Trujillo TN, Overholser BR, et al. Development and validation of a risk score to predict QT interval prolongation in hospitalized patients. Circ Cardiovasc Qual Outcomes. 2013;6(4):479-87. https://doi.org/10. 1161/CIRCOUTCOMES.113.000152.

10. Ehlert FA, Goldberger JJ, Rosenthal JE, Kadish AH. Relation between QT and RR intervals during exercise testing in atrial fibrillation. Am J Cardiol. 1992;70:332-8.

11. Ventricular Arrhythmia Risk Due to HydroxychloroquineAzithromycin Treatment For COVID-19 - American College of Cardiology. https://www.acc.org/latest-in-cardiology/articles/ 2020/03/27/14/00/ventricular-arrhythmia-risk-due-tohydroxychloroquine-azithromycin-treatment-for-covid-19.

12. Garabelli P, Stavrakis S, Albert M, et al. Comparison of QT interval readings in normal sinus rhythm between a smartphone heart monitor and a 12-Lead ECG for healthy volunteers and inpatients receiving sotalol or dofetilide. J Cardiovasc Electrophysiol. 2016;27(7):827-32. https://doi.org/10.1111/jce.12976.

Publisher's note Springer Nature remains neutral with regard to jurisdictional claims in published maps and institutional affiliations. 